\title{
Prevalencia de microsporidios y otros parásitos intestinales en pacientes con infección por VIH, Bogotá, 2001
}

\author{
Astrid Carolina Flórez 1,2, Dabeiba Adriana García ${ }^{2}$, Ligia Moncada ${ }^{3}$, Mauricio Beltrán ${ }^{4}$ \\ ${ }^{1}$ Universidad Colegio Mayor de Cundinamarca, Bogotá, D.C., Colombia. \\ ${ }^{2}$ Laboratorio de Parasitología, Instituto Nacional de Salud, Bogotá, D.C., Colombia. \\ ${ }^{3}$ Instituto de Salud Pública y Tropical, Universidad Nacional de Colombia, Bogotá D.C., Colombia. \\ ${ }^{4}$ Coordinación Nacional de Bancos de Sangre, Instituto Nacional de Salud, Bogotá, D.C., Colombia.
}

Los parásitos intestinales oportunistas son protozoos que causan diarrea en pacientes infectados con el virus de inmunodeficiencia humana $(\mathrm{VIH})$. Para determinar la prevalencia de microsporidios y otros parásitos oportunistas intestinales en pacientes infectados con el VIH con síntomas gastrointestinales y sistémicos, se estudiaron 115 pacientes que durante el 2001 consultaron al Hospital Santa Clara (33,0\%), Clínica San Pedro-ISS (20,0\%), Hospital Simón Bolívar (14,8\%), San José $(13,9 \%)$, Central de la Policía $(6,1 \%)$, Compensar $(5,2 \%)$, Liga de Lucha contra el Sida (2,6\%), Hospital San Ignacio (2,6\%) y Hospital Militar (1,7\%). La edad promedio fue de 36 años con un rango de 18 a 71 años; 14 eran mujeres y 101 hombres. La metodología empleada fue la recolección por paciente de dos muestras de materia fecal seriadas para montaje directo, concentración, coloración de Zielh Neelsen modificada para diagnóstico de coccidios intestinales y coloraciones de cromotropo modificada, Gramcromotropo y calcoflúor para diagnóstico de microsporidios. La prevalencia de oportunistas fue de 10,4 \% para Cryptosporidium sp. En cuanto a microsporidios, se encontró que de $29 \%$ de positividad con cromotropo modificada como tamizaje, tan sólo 3,5\% de las muestras se confirmaron como positivas con técnicas de calcoflúor y Gram-cromotropo. La prevalencia general de parásitos intestinales fue de $59,1 \%$, de los cuales los principales patógenos fueron Blastocystis hominis con $25,2 \%$ y Entamoeba histolytica con $13 \%$. En otros estudios con pacientes inmunosuprimidos por el VIH en Colombia, se han encontrado prevalencias de Cryptosporidium sp. menores que la hallada en esta investigación.

Palabras clave: parasitismo intestinal, infecciones oportunistas, prevalencia, síntomas, sida, microsporidios.

Prevalence of microsporidia and other intestinal parasites in HIV infected patients from Bogotá: a 2001 survey

Opportunistic intestinal parasites are a common cause of diarrhea in HIV-infected patients. To determine the prevalence of microsporidia and other opportunistic parasites infecting HIV patients in Bogotá, Colombia, 115 patients were examined for these infections during the year 2001. The institution and the sample percent from each are as follows: Santa Clara Hospital, 33.0\%; San Pedro Claver, 20.0\%; Simón Bolívar Hospital, 14.8\%; San José Hospital, 13.9\%; Central de la Policía Hospital, 6.1\%; Compensar, 5.2\%; Colombian League against AIDS, 2.6\%; San Ignacio Hospital, 2.6\%, and the Military Hospital, $1.7 \%$. The average patient age was 36 years, with a range from 18 to 71 years. Patients with complaint of gastrointestinal symptoms were asked to provide two consecutive stool samples. The samples were concentrated in formalin-ether and examined microscopically for intestinal coccidian parasites by direct wet slide mounts. The prevalence of intestinal opportunistic parasites was $10.4 \%$ for Cryptosporidium sp. Initially, $29 \%$ of the samples were found to be positive for microsporidian spores using a modified Ziehl Neelsen chromotrope stain, but only $3.5 \%$ of them were confirmed as positive when a calcofluor/Gram chromotrope stain was used. The general prevalence of intestinal parasites was $59.1 \%$. The most frequently found pathogens were Blastocystis hominis, $25.2 \%$, and Entamoeba histolytica, $13 \%$. In other studies with HIV patients in Colombia, lower prevalences of Cryptosporidium sp. infection were observed.

Key Words: intestinal parasitism, opportunistic infections, prevalence, symptoms, AIDS, microsporidia. 
Uno de los mayores problemas de salud entre los pacientes seropositivos por VIH es la infección parasitaria intestinal (1). La diarrea infecciosa de múltiples etiologías, entre las que se encuentran las producidas por parásitos como Entamoeba histolytica y Giardia duodenalis, entre otras, constituye una complicación común en sujetos infectados por el VIH, pero la infección por protozoos oportunistas representa una de las mayores infecciones de impacto significativo sobre la calidad de vida de estos pacientes (2). Se ha estimado que entre $30 \%$ y $50 \%$ de los pacientes con síndrome de inmunodeficiencia adquirida en Estados Unidos y $90 \%$ de pacientes en Haití y África sufren de diarrea crónica, y son los protozoos intestinales formadores de esporas (Cryptosporidium sp., Cyclospora sp., Isospora belli y microsporidios), los patógenos mayormente implicados en diarrea grave y síndrome de mala absorción (3). Esta infección parasitaria no es diferenciable clínicamente, pero sí a través de técnicas de laboratorio en materia fecal (4), aunque se presenta una gran dificultad en el diagnóstico de microsporidiosis debido al tamaño de las esporas de las diferentes especies que infectan el ser humano, el cual varía entre 1,0 y 1,5 por 4,0 $\mu \mathrm{m}$ (5). El aislamiento de estos parásitos en pacientes con sida y diarrea crónica varía entre $40 \%$ y $83 \%$, pero difiere sensiblemente de región a región (4).

Otros protozoos han sido incriminados con menos frecuencia como causa de enfermedad diarreica en pacientes inmunocomprometidos. Entre estos están G. duodenalis, Trichomona hominis, Balantidium coli, E. histolytica, Strongyloides stercoralis $(4,6)$ y Blastocystis hominis, cuyo papel como patógeno humano ha sido controvertido. Sin embargo, algunas evidencias sugieren que es un agente implicado en causar diarrea en pacientes inmunosuprimidos, especialmente cuando está presente en gran cantidad $(7,8)$.

Correspondencia:

Astrid Carolina Flórez, Laboratorio de Parasitología, Instituto Nacional de Salud, Av.Calle 26 No. 51 60, Bogotá, D.C., Colombia

Teléfono: 220-7700, extensión 454

aflorez@ins.gov.co

Recibido: 31/01/03; aceptado: 23/05/03
La enfermedad gastrointestinal en pacientes seropositivos por VIH con síntomas como diarrea, deshidratación, disfagia, dolor abdominal, ictericia, pérdida de peso y sangrado gastrointestinal puede ser el resultado de la infección oportunista (9).

En el presente estudio se determinó la prevalencia de microsporas y otros parásitos intestinales y su relación con síntomas gastrointestinales y sistémicos en pacientes con infección con VIH, pertenecientes al programa de enfermedades infecciosas de diferentes centros hospitalarios de Bogotá.

\section{Materiales y métodos}

\section{Población de estudio}

Durante el período comprendido entre enero y diciembre del 2001 fueron incluidos en el estudio 115 pacientes con infección por VIH. Se aplicó una encuesta para recoger información sobre datos personales, tendencia sexual, prueba de ELISA y confirmación por Western blot, tratamiento antirretroviral y la de uno o más síntomas gastrointestinales o sistémicos que incluían diarrea, dolor abdominal, vómito, náuseas, aumento del peristaltismo, anorexia, pérdida de peso y fiebre durante el tiempo de recolección de las muestras de materia fecal. Además, se solicitó el debido consentimiento informado a cada paciente para la utilización de sus muestras en el estudio, acogiéndose a las normas sobre aspectos éticos de investigación en humanos de la Resolución 008430 del Ministerio de Salud.

\section{Muestras}

De cada paciente se recolectaron dos muestras de materia fecal seriadas con un tiempo no mayor a 10 días entre muestra y muestra. Las muestras fueron procesadas dentro de las 3 horas siguientes a su recolección.

\section{Procesamiento de muestras}

Las muestras se estudiaron con preparaciones en solución salina y solución de yodo hechas directamente y del sedimento producto de la concentración por la técnica de sedimentación formol-éter, de acuerdo con Ritchie (10). Para la identificación de E. histolytica se valoró la presencia del cariosoma central de los trofozoítos 
con azul de metileno amortiguado y la presencia de eritrofagocitosis por la visualización microscópica de trofozoítos con glóbulos rojos fagocitados como indicadores de una amibiasis aguda invasora (11). Para la identificación de coccidios intestinales (Cryptosporidium sp., Cyclospora sp. e I. belli), los sedimentos fueron teñidos con la coloración ácido resistente de ZiehlNeelsen modificada (12) y para los microsporidios se utilizó la coloración de cromotropo modificada, en la cual las esporas tienen una alta afinidad por el cromotropo $2 \mathrm{R}$, tomando una coloración de rosado a rojo que se acentúa en la pared y en su interior en forma de banda como un cinturón; la técnica de Gram-cromotropo según Moura, en la cual las esporas de microsporidios retienen el cristal violeta y muestran dos tipos de características en su coloración y pueden exhibir una notable positividad del Gram o poseer distintos gránulos Gram positivos $(13,14)$, y la técnica fluorocrómica con calcoflúor según Didier, que tiene afinidad por la quitina ubicada en la endospora de los microsporidios (5).

\section{Análisis estadístico}

Para las variables cuantitativas se utilizó la media aritmética, la mediana y la desviación estándar. La prevalencia de parásitos se estimó mediante proporciones de prevalencia general. Para establecer si existía relación entre la prevalencia de parásitos y los diferentes síntomas de la población en estudio, se realizaron pruebas estadísticas de diferencias de proporciones. Para este análisis se empleó el programa Epilnfo versión 6,04 (15).

\section{Resultados}

Participaron en el estudio 115 pacientes, cuya procedencia fue la siguiente: Hospital Santa Clara, 33\%; Clínica San Pedro del Seguro Social, 20\%; Hospital Simón Bolívar, 14,8\%; Hospital San José, 13,9\%; Clínica Central de la Policía, 6,1\%; Compensar EPS, 5,2\%; Liga de Lucha contra el Sida, 2,6\%; Hospital San Ignacio, 2,6\%, y Hospital Militar, 1,7\%.

La edad promedio de los 115 pacientes fue de 36 años (rango 18-71 años); 14 (12,2\%) eran mujeres y $101(87,8 \%)$ hombres, de los cuales 43 manifestaron ser homosexuales.
Del total de pacientes, $36(31,3 \%)$ estaban hospitalizados en el momento de la recolección de las muestras de materia fecal, 79 (68,7\%) eran ambulatorios y $68(59,1 \%)$ se encontraban con tratamiento antirretroviral.

La prevalencia de parásitos en los 115 pacientes fue de $59,1 \%$ correspondiente a 68 pacientes, de los cuales $19(27,9 \%)$ presentaron más de un parásito o poliparasitismo. En los 68 pacientes parasitados se observó que 58 (85,3\%) presentaron parásitos patógenos y los restantes no patógenos. El más prevalente de los parásitos con potencial patógeno fue $B$. hominis $(25,2 \%)$, seguido de $E$. histolytica con $13 \%$, cuyo hallazgo fue confirmado con la identificación de trofozoítos y la presencia de eritrofagocitosis; otros patógenos encontrados fueron $G$. duodenalis con $1,8 \%$ e Hymenolepis nana con 0,9\%. En 20 $(17,5 \%)$ de estos pacientes se demostraron parásitos considerados como oportunistas, Cryptosporidium sp. en 12 pacientes (10,5\%), 4 pacientes $(3,5 \%)$ con especies de microsporidios y $3,5 \%$ con Strongyloides stercoralis.

En el hallazgo de otros parásitos no patógenos o cuyo poder patógeno no fue confirmado, la frecuencia más alta $(15,6 \%)$ correspondió a Endolimax nana, seguida de Entamoeba coli $(4,3 \%)$, lodamoeba butschlii $(4,3 \%)$, quistes compatibles con el complejo Entamoeba histolytica/Entamoeba dispar (4,3\%) y Entamoeba hartmanni $(2,6 \%)$.

El grupo de edad de 26 a 35 años mostró el mayor porcentaje de positividad parasitaria con 56,5\% de parasitismo por parásitos patógenos y el grupo de pacientes mayor de 56 años presentó el menor porcentaje de positividad parasitaria, con $25 \%$ de parásitos patógenos y ausencia de parásitos no patógenos.

De acuerdo con la manifestación de diarrea en los pacientes infectados por el VIH con presencia de parásitos patógenos, se observó que de 66,6\% a $100 \%$ presentaba diferentes episodios de diarrea con una media de 5 evacuaciones por día (cuadro 1). Aquellos pacientes que manifestaron el síntoma de diarrea tuvieron mayor tendencia a presentar parásitos patógenos $(p<0,05)$ (cuadro 2). 
Cuadro 1. Parásitos intestinales detectados en pacientes infectados con el VIH y su correlación con diarrea.

\begin{tabular}{|c|c|c|}
\hline Parásitos patógenos detectados & $\begin{array}{c}\text { Pacientes } \\
\text { parasitados } \\
n=58\end{array}$ & $\begin{array}{l}\text { Pacientes con } \\
\text { diarrea } \\
(\%)\end{array}$ \\
\hline Cryptosporidium sp. & 8 & 100 \\
\hline Cryptosporidium sp. - Entamoeba coli & 1 & 0 \\
\hline Cryptosporidium sp. - Blastocystis hominis & 1 & 100 \\
\hline Cryptosporidium sp. - Entamoeba histolytica & 1 & 100 \\
\hline Blastocystis hominis - Endolimax nana & 1 & 100 \\
\hline \multicolumn{3}{|l|}{ Entamoeba histolytica/Entamoeba dispar - Cryptosporidium sp. } \\
\hline Blastocystis hominis & 15 & 80 \\
\hline Blastocystis hominis - Strongyloides stercoralis & 2 & 50 \\
\hline Blastocystis hominis - Endolimax nana - Entamoeba hartmanni & 1 & 100 \\
\hline \multicolumn{3}{|l|}{ Blastocystis hominis - Endolimax nana - Entamoeba coli } \\
\hline Entamoeba histolytica/Entamoeba dispar & 1 & 100 \\
\hline Blastocystis hominis - Entamoeba histolytica & 2 & 100 \\
\hline Blastocystis hominis - Giardia duodenalis & 1 & 100 \\
\hline Blastocystis hominis - Endolimax nana - Hymenolepis nana & 1 & 100 \\
\hline \multicolumn{3}{|l|}{ Blastocystis hominis - lodamoeba butschlii } \\
\hline Entamoeba histolytica/Entamoeba dispar & 1 & 100 \\
\hline Blastocystis hominis - Endolimax nana & 1 & 100 \\
\hline \multicolumn{3}{|l|}{ Blastocystis hominis - Endolimax nana - Entamoeba hartmanni } \\
\hline Entamoeba histolytica/Entamoeba dispar & 1 & 0 \\
\hline Blastocystis hominis - Endolimax nana - Entamoeba coli & 1 & 0 \\
\hline Giardia duodenalis & 1 & 100 \\
\hline Entamoeba histolytica & 7 & 100 \\
\hline \multicolumn{3}{|l|}{ Entamoeba coli - Endolimax nana - Iodamoeba butschlii - } \\
\hline Entamoeba histolytica & 1 & 100 \\
\hline Endolimax nana - Entamoeba histolytica & 2 & 100 \\
\hline \multicolumn{3}{|l|}{ Endolimax nana - lodamoeba butschilii - } \\
\hline Entamoeba histolytica/Entamoeba dispar & 1 & 100 \\
\hline Strongyloides stercoralis & 2 & 0 \\
\hline Microsporidios & 3 & 66,6 \\
\hline Microsporidios - Entamoeba histolytica & 1 & 100 \\
\hline Iodamoeba butschilii - Entomoeba histolytica & 1 & 100 \\
\hline TOTAL & 58 & \\
\hline
\end{tabular}

Cuadro 2. Diarrea en pacientes infectados con el VIH con y sin parásitos intestinales.

\begin{tabular}{|c|c|c|c|c|c|c|}
\hline Síntomas & $\begin{array}{c}\text { Positivos para } \\
\text { parásitos } \\
n=68\end{array}$ & $\begin{array}{c}\text { Negativos para } \\
\text { parásitos } \\
n=47\end{array}$ & $\begin{array}{l}\text { Total } \\
\mathrm{n}=115\end{array}$ & $\mathbf{R P}^{*}$ & IC95\% & $p$ \\
\hline \multicolumn{7}{|l|}{ Diarrea } \\
\hline Sí & 58 & 32 & 90 & 1,61 & $0,97-2,67$ & $<0,05$ \\
\hline No & 10 & 15 & 25 & & & \\
\hline Síntomas & $\begin{array}{l}\text { Presencia de } \\
\text { parásitos } \\
\text { patógenos } \\
n=58\end{array}$ & $\begin{array}{c}\text { Presencia de } \\
\text { parásitos } \\
\text { no patógenos } \\
n=10\end{array}$ & $\begin{array}{l}\text { Total } \\
\mathrm{n}=68\end{array}$ & $\mathbf{R P}^{*}$ & IC95\% & $p$ \\
\hline \multicolumn{7}{|l|}{ Diarrea } \\
\hline Sí & 48 & 4 & 54 & 1,48 & $1,00-2,18$ & $<0,05$ \\
\hline No & 10 & 6 & 14 & & & \\
\hline
\end{tabular}

*RP: razón de prevalencia o positividad (riesgo de un individuo de ser positivo y presentar un síntoma) 
Sin embargo, con respecto a los demás síntomas incluidos en la encuesta, se realizó un análisis con 104 pacientes, quienes tenían información completa en sus historias clínicas o respondieron a las preguntas en su totalidad. Ninguno de los síntomas evaluados fue estadísticamente significativo al correlacionarlo directamente con la presencia de parásitos intestinales, pero el mayor riesgo de ser positivo a la infección por parásitos intestinales y presentar algún síntoma gastrointestinal asociado se manifestó con el dolor abdominal y la presencia de fiebre (razón de prevalencia o positividad, $\mathrm{RP}=1,33$; IC95\%=0,95 $-1,88$ y $R P=1,35 ;$ IC $95 \%=1,01-1,81$ ) (cuadro 3).

Se halló una prevalencia de 7,0\% de E. histolytica y quistes compatibles con el complejo E.histolytica/E.dispar en pacientes con tendencia homosexual. Sin embargo, no se observó una asociación estadísticamente significativa $(\mathrm{RP}=1,09 ; \mathrm{IC} 95 \%=0,60-1,97 ; p>0,05)$.

\section{Discusión}

En este estudio se evaluó la prevalencia de microsporidios y otros parásitos intestinales, incluidos los oportunistas, en 115 pacientes infectados con el VIH procedentes de diferentes centros hospitalarios de Bogotá, en donde estas cifras aún son desconocidas. Además, teniendo en cuenta que los síntomas gastrointestinales son complicaciones frecuentes de la infección por VIH (16), se investigó la asociación entre la identificación de parásitos y la presencia de algunos síntomas gastrointestinales y sistémicos como diarrea, anorexia, dolor abdominal, vómito, náuseas, aumento del peristaltismo, pérdida de peso y fiebre.

En Colombia son escasos los estudios de parasitismo en pacientes positivos para $\mathrm{VIH}$; en 1999 se determinó la frecuencia de parásitos intestinales en pacientes positivos para VIH en Bogotá, y se encontraron como patógenos de mayor frecuencia $G$. duodenalis con $4,7 \%$ y $E$. histolytica con 2,8\%; otros oportunistas como $I$. belli se halló en $1,9 \%$, S. stercoralis, en $0,9 \%$, Cryptosporidium sp., en $0,9 \%$ y no se encontró Cyclospora sp. (17). No obstante, en comparación con este estudio, las cifras de prevalencia obtenidas fueron mayores para parásitos oportunistas como Cryptosporidium sp. con 10,5\%,

Cuadro 3. Otros síntomas en pacientes infectados con el VIH con y sin parásitos intestinales.

\begin{tabular}{|c|c|c|c|c|c|c|}
\hline Síntomas & $\begin{array}{c}\text { Positivos para } \\
\text { parásitos } \\
n=64\end{array}$ & $\begin{array}{l}\text { Negativos para } \\
\text { parásitos } \\
n=40\end{array}$ & Total & $\mathbf{R P}^{*}$ & IC95\% & $p$ \\
\hline \multicolumn{7}{|l|}{ Anorexia } \\
\hline Sí & 29 & 16 & 45 & 1,09 & $0,80-1,47$ & 0,742 \\
\hline No & 35 & 24 & 59 & & & \\
\hline \multicolumn{7}{|c|}{ Dolor abdominal } \\
\hline Sí & 43 & 20 & 63 & 1,33 & $0,95-1,88$ & 0,123 \\
\hline No & 21 & 20 & 11 & & & \\
\hline \multicolumn{7}{|l|}{ Vómito } \\
\hline Sí & 17 & 11 & 28 & 0,98 & $0,69-1,39$ & 0,902 \\
\hline No & 47 & 29 & 76 & & & \\
\hline \multicolumn{7}{|l|}{ Náuseas } \\
\hline Sí & 37 & 21 & 58 & 1,09 & $0,80-1,48$ & 0,743 \\
\hline No & 27 & 19 & 46 & & & \\
\hline \multicolumn{7}{|c|}{ Aumento del peristaltismo } \\
\hline Sí & 43 & 30 & 73 & 0,87 & $0,64-1,18$ & 0,530 \\
\hline No & 21 & 10 & 31 & & & \\
\hline \multicolumn{7}{|c|}{ Disminución de peso } \\
\hline Sí & 44 & 28 & 72 & 0,98 & $0,71-1,35$ & 0,933 \\
\hline No & 20 & 12 & 32 & & & \\
\hline \multicolumn{7}{|l|}{ Fiebre } \\
\hline Sí & 28 & 10 & 38 & 1,35 & $1,01-1,81$ & 0,084 \\
\hline No & 36 & 30 & 66 & & & \\
\hline
\end{tabular}

*RP: razón de prevalencia o positividad (riesgo de un individuo de ser positivo y presentar un síntoma) 
especies de microsporidios, 3,5\% y $S$. stercoralis, $3,5 \%$, posiblemente debido al crecimiento de la epidemia de sida y a la calidad de vida que ha ido disminuyendo para la mayoría de estos pacientes, tanto por el alto costo del tratamiento antirretroviral como por la falta de adherencia o el abandono del tratamiento. Además, aunque tuvimos como limitante el desconocimiento del verdadero nivel de inmunosupresión de la mayoría de los pacientes a través del conteo de linfocitos T CD4+, muchos de ellos se encontraban en un estadio de la enfermedad muy avanzado, si tenemos en cuenta que 8 de los 12 pacientes con Cryptosporidium sp. se hallaban hospitalizados. Sin embargo, I. belli y Cyclospora sp. no se hallaron, posiblemente porque $59,1 \%$ de los pacientes se encontraba con tratamiento antirretroviral, cuyo esquema incluye el trimetroprim-sulfametoxazol como profilaxis para algunas infecciones, incluidos estos dos coccidios intestinales (18). Otros estudios describen la Cyclospora sp. como un patógeno oportunista presente en grupos de personas inmunosuprimidas y turistas o residentes extranjeros en países en desarrollo $(19,20)$, pero en Colombia sólo se han descrito 3 casos de ciclosporidiasis en pacientes inmunocompetentes (21).

La prevalencia de E. histolytica fue de $13 \%$, mayor a la de 2,8\% encontrada en el estudio de López y col. (17) en pacientes colombianos, pero similar a otros estudios como el realizado en India, donde la prevalencia fue de $14,9 \%$, lo que sugiere la práctica de malos hábitos higiénicos en individuos infectados por el $\mathrm{VIH}$; de otro lado, en este estudio se obtuvo una prevalencia de $7,0 \%$ de $E$. histolytica y complejo E.histolytica/E.dispar en pacientes con tendencia homosexual y $23,6 \%$ en pacientes sin esta tendencia; la prevalencia en homosexuales fue un poco mayor que la informada en estudios realizados en Estados Unidos y Canadá, donde entre 1970 y 1980 se documenta que, al menos, $30 \%$ de los hombres homosexuales se encontraban infectados con especies de Entamoeba debido a sus prácticas sexuales. Más recientemente esta prevalencia ha disminuido sensiblemente según otros estudios y se han obtenido cifras de tan sólo 3,3\%, lo que refleja la utilización de más y mejores medidas preventivas, lo cual ha reducido el factor de riesgo en las prácticas sexuales entre homosexuales (22). Al igual que otros parásitos intestinales, E. histolytica se ha determinado como de mayor prevalencia en personas homosexuales debido al alto riesgo en sus diferentes prácticas sexuales (11), situación que debe ser analizada en conjunto con el tipo de prácticas sexuales en estos pacientes para establecer una posible asociación en nuestro medio.

Con respecto a la prevalencia de $S$. stercoralis $(3,5 \%)$, cabe anotar que fue mayor a la encontrada en el estudio de López y col. (0,9\%) (17), pero similar a otros estudios realizados en Brasil (2,5\%) (23), India (5,3\%) (24), Africa y Asia, donde más y más casos de estrongiloidosis se han informado durante los últimos años (25). Otros patógenos como G. duodenalis, cuya prevalencia de 1,8\% fue baja en relación con la hallada por López y col. $(4,7 \%)(17)$, menor en comparación con otros estudios desarrollados en India (4,3\%) (24) y Tailandia $(3,8 \%)(26)$, pero mayor que la de otros estudios realizados también en pacientes con VIH en India (8,3\%) (27) y Brasil (16\%) (23). Sin embargo, a pesar de su escasa prevalencia es importante destacar que en países fronterizos como Brasil se ha concluido que la estrongiloidosis y la giardiosis son más frecuentes en pacientes infectados por el $\mathrm{VIH}$, como lo demostró un estudio retrospectivo realizado en Bahía (28). Los hallazgos de nuestro estudio, junto con un caso de himenolepiasis por Hymenolepis nana en uno de los pacientes, nos hace pensar que algunos parásitos, considerados como patógenos en la actualidad, se comportan como oportunistas y día tras día han ido incrementando en pacientes inmunodeprimidos.

En cuanto a las prevalencias de los parásitos no patógenos hallados, como E. nana (16\%) y $I$. butschlii $(4,3 \%)$, los resultados fueron muy similares al estudio de López y col. (17), pero comparados con los de otros estudios realizados en población general, como la Encuesta Nacional de Morbilidad desarrollada en Colombia en 1966 y el Estudio Nacional de Salud en 1980, las prevalencias de estos parásitos no patógenos fueron menores; así, E. nana se encontró con prevalencias de $34,1 \%$ y $49,6 \%$, respectivamente, 
en tanto que en estudios hechos en Brasil se encontró con 3,5\% (25). En el caso de I. butschlii, las prevalencias fueron de $7,7 \%$ y $17 \%$, respectivamente, y de $0,3 \%$ en estudios realizados en Tailandia (26). Con respecto a E. coli, otro de los parásitos intestinales no patógenos encontrados en el estudio, se halló una prevalencia de $4,3 \%$, que es intermedia entre la de $0,8 \%$ informada por Monadas et al. (27) y la del $13 \%$ encontrada por Cimerman et al. (23). Sin embargo, se piensa que aunque las condiciones higiénicas han mejorado, la presencia de estos parásitos no patógenos constituye un indicador de contaminación fecal en estos individuos, posiblemente debida no solamente a malos hábitos higiénicosanitarios, sino a otros factores de riesgo incluidas las diferentes prácticas sexuales $(4,22,26,29,30)$.

Con relación a la prevalencia de infección por especies de microsporidios, en el estudio de López y col. (17) no hubo hallazgo y en nuestro estudio se demostró una baja prevalencia, representada en $4 / 115(3,5 \%)$ pacientes, de los cuales 3 presentaban diarrea. Debido al método de diagnóstico de microscopia de luz utilizado, posiblemente exista una sobrestimación de la prevalencia de microsporidios reflejada en la dificultad que se presenta en la identificación de las esporas debido a su tamaño, que varía entre $1,5 \mu \mathrm{m}$ a 2,0 $\mu \mathrm{m}$ por $4,0 \mu \mathrm{m}(5,17)$, así como la problemática diferenciación de las esporas de otros microorganismos presentes en la materia fecal y, probablemente, debida también a la variabilidad geográfica, que genera diferentes factores de riesgo para la exposición a la infección por microsporidios, presentes en otros países donde las prevalencias varían entre 7\% y 50\% (16). Es importante resaltar que la prevalencia de microsporidiosis intestinal en el presente estudio se halló de manera general a través de la confirmación de la presencia de esporas mediante coloraciones especiales que no brindan la posibilidad de determinar especies de microsporidios, cuyo criterio de identificación está basado en estructuras internas de la espora visualizadas tan sólo por microscopia electrónica o por la aplicación de técnicas con anticuerpos monoclonales (16). Sin embargo, con respecto a las tres técnicas de coloración empleadas en este estudio, cabe anotar que la técnica de cromotropo modificada tiene el inconveniente de que algunas bacterias y levaduras pueden tomar la coloración rojiza del cromotropo $2 \mathrm{R}$, efecto que puede aminorarse con la coloración de Gram-cromotropo, que acentúa mucho más las características de las esporas, lo cual facilita su detección aunque estén en pequeñas cantidades; en cuanto al tercer método por calcoflúor, su afinidad por la quitina bajo la luz ultravioleta diferencia más fácilmente las esporas de microsporidios, aun en aquellas zonas más espesas de la muestra, lo cual mejora la sensibilidad de la técnica; por esta razón, la prevalencia total de microsporidiosis fue confirmada por los métodos de Gram-cromotropo y calcoflúor.

Con respecto a $B$. hominis, existe evidencia de que se trata de un protozoo asociado con la presencia de diarrea en pacientes con sida $(7,31)$. Aunque el papel de $B$. hominis como patógeno emergente aún permanece sin resolver totalmente, en este estudio se tuvo en cuenta el criterio diagnóstico de algunas investigaciones, en las cuales la presencia de más de 5 parásitos por campo microscópico con objetivo de $40 \mathrm{X}$ se considera positivo; este número de parásitos excretados en materia fecal puede ser un indicador del papel patogénico en pacientes positivos para VIH con diarrea (4,11). En 1998 y 1999, dos estudios de investigación en 100 venezolanos y 1.216 italianos demostraron que sólo en sujetos con inmunosupresión, $B$. hominis mostraba una asociación significativa con síntomas gastrointestinales; por tanto, se sugirió su papel patogénico como oportunista $(32,33)$. En esta población de pacientes infectados con el VIH se demostró una prevalencia de $25,2 \%$, correspondiente a 29 pacientes de 115, de los cuales 15 mostraron $B$. hominis como único microorganismo, $80 \%$ de ellos con diarrea, y en 14 de ellos asociado con otros parásitos.

El grupo de 26 a 35 años mostró mayor prevalencia de parásitos patógenos; este grupo de edad es precisamente el rango en que una persona es laboralmente más productiva, pero a causa de la infección por el VIH/sida y sus complicaciones como la enfermedad diarreica y otros síntomas gastrointestinales se puede presentar una gran 
disminución en su calidad de vida, sumado al hecho del profundo rechazo que pueden recibir dentro de su núcleo de convivencia laboral y familiar (34).

En algunos estudios en los que se ha investigado la asociación entre síntomas gastrointestinales y la presencia de parásitos patógenos intestinales, generalmente se ha tenido en cuenta la relación entre el estado inmunológico del paciente infectado con el VIH, el cual se considera inmunocomprometido por un recuento total de linfocitos T CD4+ menor a 100 células/ $\mu \mathrm{l}$, asociado con un mayor riesgo de adquirir infección por parásitos intestinales, especialmente oportunistas y con la presencia de síntomas como la diarrea. Sin embargo, la causa de la diarrea en pacientes infectados por el VIH puede ser de tipo inexplicable y aún estar asociada con los medicamentos antirretrovirales $(1,16,34,35)$. En el presente estudio, no se pudo evaluar esta asociación, debido al desconocimiento del recuento de linfocitos $T$ CD4+ y, por tanto, del estado inmune de estos pacientes. Sin embargo, de acuerdo con la valoración de la consistencia de las dos muestras de materia fecal recogidas por paciente, con los datos suministrados por las historias clínicas en el caso de los hospitalizados y con la encuesta realizada al paciente ambulatorio, se observó que existe una tendencia a encontrar mayor número de pacientes con parásitos intestinales tanto patógenos como no patógenos en presencia del síntoma de diarrea; sin embargo, a pesar de que los estimados de riesgo ( $R P=1,61$; IC95\%: 0,97$2,67)$ y $(\mathrm{RP}=1,48$; IC95\%: 1,0-2,18) podrían indicar esta asociación, la inclusión de pacientes sin síntomas gastrointestinales permitiría definir este hallazgo (cuadro 2).

Finalmente, con relación a otros síntomas presentes en estos pacientes con infección parasitaria, la asociación no fue estadísticamente significativa para ninguno de ellos, pero la fiebre y el dolor abdominal fueron los síntomas que mostraron el mayor riesgo de estar presentes en la infección parasitaria intestinal en este grupo de individuos.

Es importante tener en cuenta que el diagnóstico de algunos de los parásitos oportunistas intestinales con la técnica de Ziehl Neelsen modificada, constituye una herramienta sencilla y al alcance tanto del médico tratante como de los laboratorios clínicos de cualquier nivel, a través de la cual se beneficiará el paciente infectado con el VIH mediante un tratamiento oportuno de estos parásitos, y disminuirá su morbilidad y posible mortalidad asociada.

\section{Agradecimientos}

Los autores expresan sus agradecimientos a todos los pacientes consultantes de las unidades de Infectología de los hospitales Santa Clara, Simón Bolívar, San José, San Ignacio y Militar, las clínicas San Pedro Claver, ISS, Central de la Policía, a Compensar, EPS y la Liga de Lucha contra el Sida, así como también a todos los profesionales, médicos, infectólogos y enfermeras, en especial a Ana María Granados del Hospital Santa Clara, a Luis Yela Yela del Seguro Social, Seccional Cundinamarca, por su gran colaboración para el desarrollo de este proyecto.

\section{Referencias}

1. Wiwanitkit V. Intestinal parasitic infections in Thai HIVinfected patients with different immunity status. BMC Gastroenterology 2001;1:3.

2. Brandonisio $O$, Maggy $\mathbf{P}$, Panaro MA, Lisi S, Andriola A, Acquafredda A, et al. Intestinal protozoa in HIV- infected in Abulia, South Italy. Epidemiol Infect 1999;123:457-62.

3. Xiao L, Lal R, Lal A. Effect of immune activation induced by Cryptosporidium parvum whole antigen on in vitro human immunodeficiency virus type 1 infection. J Infect Dis 1999;180:559-63.

4. Prasad KN, Nag VL, Dhole TN, Ayyagari A. Identification of enteric pathogens in HIV-positive patients with diarrhoea in Nothern India. J Health Popul Nutr 2000;18:23-6.

5. Didier E, Orenstein J, Aldras A, Bertucci D, Rogers L, Janney A. Comparison of three staining methods for detecting microsporidia in fluids. J Clin Microbiol 1995; 33:3138-45.

6. Ferreira Marcelo $\mathbf{S}$. Infections by protozoa in immunocompromised host. Mem Inst Oswaldo Cruz 2000;95:159-62.

7. Brites C, Barberino MG, Bastos MA, Sampaio SaM, Silva N. Blastocystis hominis as a potential cause of diarrhea in AIDS patients: a report of six cases in Bahia, Brazil. Braz J Infect Dis 1997;2:91-4.

8. Ok UZ, Girginkardesler N, Balcioglu C, Ertan P, Pirildar T, Kilimcioglu AA. Effect of trimethoprim- 
sulfamethaxazole in Blastocystis hominis infection. Am J Gastroenterol 1999;94:3245-7.

9. Sievert W, La Brooy JT. HIV-related gastrointestinal disease. Med J Aust 1993;158:175-8.

10. Ridley DS, Hawgood BC. The value of formol-ether concentration of faecal cysts and ova. J Clin Pathol 1956;9:74-6.

11. Atías A. Parasitología médica. Santiago de Chile: Publicaciones Técnicas Mediterráneo Ltda.; 1997.

12. Casemore DP, Armstrong M, Sands RL. Laboratory diagnosis of cryptosporidiosis. J Clin Pathol 1985;38: 1337-41.

13. Moura H, Nunes da Silva J, Sodre F, Brasil P, Wallmo $\mathrm{K}$, Wahlquist $\mathrm{S}$, et al. Gram-chromotrope: a new technique that enhances detection of microsporidial spores in clinical samples. J Euk Microbiol 1996; 43:94-5.

14. Moura H, Schwartz D, Bornay-Llinares F, Sodre F, Wallace S, Visvesvara G. A new improved "quick-hot Gram-chromotrope" technique that differentially stains microsporidian spores in clinical samples, including paraffin-embedded tissues sections. Arch Pathol Lab Med 1997;121:888-93.

15. CDC, Center For Disease Control and Prevention. U.S.A. Epi Info 6, Versión 6.04. World Health Organization Geneva, Switzerland; 1996.

16. Sobottka I, Schwartz D, Schottelius J, Visvesvara G, Pieniazek N, Schmetz C, et al. Prevalence and clinical significance of intestinal microsporidiosis in human immunodeficiency virus-infected patients with and without diarrhea in Germany: a prospective coprodiagnostic study. Clin Infect Dis 1998;26:475-80.

17. López C, Moncada L, Murcia M, Saravia J, Nicholls RS. Frecuencia de parásitos intestinales en pacientes $\mathrm{VIH}+$ en la ciudad de Santa Fe de Bogotá. Rev Fac Med UN Col 1999;47:9-12.

18. Pape JW, Verdier RI, Boncy M, Boncy J, Johnson WD. Cyclospora adults infected with HIV, clinical manifestations, treatment, and prophylaxis. Ann Intern Med 1994;121:654-7.

19. Ashford RW. Ocurrence of an undescribed coccidian in man in Papua. New Guinea. Ann Trop Med Parasitol 1979;73:497-500.

20. Soave R, Dubey JP, Ramos LJ, Tummings M. A new intestinal pathogen? Clin Res 1986;34:533 A.

21. Agudelo P, Restrepo M, Galvis MT, Botero D. Infección por Cyclospora sp. en tres pacientes inmunocompetentes. Biomédica 2000;20:25-32.

22. Reed SL. Entamoeba infections in human immunodeficiency virus-infected patients: not just a tropical problem. Clin Infect Dis 2000;30:959-61.
23. Cimerman S, Cimerman B, Lewi DS. Prevalence of intestinal parasitic infections in patients with acquired immunodeficiency sindrome in Brazil. Int $\mathrm{J}$ Infect Dis 1999;3:203-6.

24. Joshi M, Chowdhary AS, Dalal PJ, Maniar JK. Parasitic diarrhoea in patients with AIDS. Natl Med $\mathrm{J}$ India 2002;15:72-4.

25. Singh S. Human strongyloidiasis in AIDS era: its zoonotic importance. J Assoc Physicians India 2002;50:415-22.

26. Waywa D, Kongkriengdaj S, Chaidatch S, Tiengrim $\mathrm{S}$, Kowadisaibura $\mathrm{B}$, Chaikachonpat $\mathrm{S}$, et al. Protozoan enteric infection in AIDS related diarrhea in Thailand. Southeast Asian J Trop Med Public Health 2001;32:151-5.

27. Mohandas K, Sehgal R, Archane S, Malla N. Prevalence of intestinal parasitic pathogens in HIVseropositive individuals in Northern India. Jpn $\mathrm{J}$ Infect Dis 2002;55:83-4.

28. Feitosa G, Bandeira AC, Sampaio DP, Badaro R, Brites C. High prevalence of giardiasis and strongyloidiasis among HIV-infected patients in Bahia, Brazil. Braz J Infect Dis 2001;5:339-44.

29. Gassama A, Sow PS, Fall F , Camara P, GueyeNdiaye A, Seng $R$, et al. Ordinary and opportunistic enteropathogens associated with diarrhea in Senegalese adults in relation to human immunodeficiency virus serostatus. Int $\mathrm{J}$ Infect Dis 2001; 5:192-8.

30. Corredor A, Arciniegas E, Hernández CA, Cáceres E, Castaño L, Estupiñan D, et al. Parasitismo intestinal. Santafé de Bogotá, D.C.: Instituto Nacional de Salud; 2000.

31. Albrecht H, Stellbrink HJ, Koperski K, Greten H. Blastocystis hominis in human immunodeficiency virusrelated diarrhoea. Scand J Gastroenterol 1995; 30: 909-14.

32. Devera R, Azacon B, Jimenez M. Blastocystis hominis in patiens at the Ruiz y Paez University Hospital from Bolivar City, Venezuela. Bol Chil Parasitol 1998; 53:6570.

33. Cirioni O, Giacometti A, Drenaggi D. Prevalence and clinical relevance of Blastocystis hominis in diverse patient cohorts. Eur J Epidemiol 1999;15:389-93.

34. Wanke C, Cohan D, Thummakul T, Jongwuitiwes S, Grayson L, Hammer S, Hanvanich M. Diarrheal Disease in patients infected with human immunodeficiency virus in Bangkok, Thailand. Am J Trop Med Hyg 1999;60:871-4.

35. Monkemuller KE, Wilcox CM. Investigation of diarrhea in AIDS. Can J Gastroenterol 2000;14:933-40. 